\title{
Concomitant Medication End Date
}

National Cancer Institute

\section{Source}

National Cancer Institute. Concomitant Medication End Date. NCI Thesaurus. Code C83136.

The stop date of the concomitant medication. 